\title{
Implementation of Real-Time Laboratory-Based Influenza Surveillance System, Thailand
}

\author{
Phunlerd Piyaraj ${ }^{\star 1,2}$, Nira Pet-hoi ${ }^{3}$, Chaiyos Kunanusont ${ }^{2}$, Supanee Sangiamsak ${ }^{2}$, \\ Somsak Wankijcharoen ${ }^{3}$, Jarupa Kanjanakornhirun ${ }^{3}$ and Paithoon Boonma ${ }^{2}$
}

${ }^{1}$ Phramongkutklao College Of Medicine, Bangkok, Thailand; ${ }^{2}$ Bangkok Health Research Center, Bangkok Dusit Medical Services Public Company Limited (BDMS), Bangkok Hospital Group, Bangkok, Thailand; ${ }^{3}$ Chief Information Office, Bangkok Hospital Head Quarter, Bangkok, Thailand

\section{Objective}

We describe the Bangkok Dusit Medical Services Surveillance System (BDMS-SS) and use of surveillance efforts for influenza as an example of surveillance capability in near real-time among a network of 20 hospitals in the Bangkok Dusit Medical Services group (BDMS).

\section{Introduction}

Influenza is one of the significant causes of morbidity and mortality globally. Previous studies have demonstrated the benefit of laboratory surveillance and its capability to accurately detect influenza outbreaks earlier than syndromic surveillance. ${ }^{1-3}$ Current laboratory surveillance has an approximately 4-week lag due to laboratory test turn-around time, data collection and data analysis. As part of strengthening influenza virus surveillance in response to the 2009 influenza A (H1N1) pandemic, the real-time laboratorybased influenza surveillance system, the Bangkok Dusit Medical Services Surveillance System (BDMS-SS), was developed in 2010 by the Bangkok Health Research Center (BHRC). The primary objective of the BDMS-SS is to alert relevant stakeholders on the incidence trends of the influenza virus. Type-specific results along with patient demographic and geographic information were available to physicians and uploaded for public health awareness within 24 hours after patient nasopharyngeal swab was collected. This system advances early warning and supports better decision making during infectious disease events. ${ }^{2}$ The BDMS-SS operates all year round collecting results of all routinely tested respiratory clinical samples from participating hospitals from the largest group of private hospitals in Thailand.

\section{Methods}

The BDMS has a comprehensive network of laboratory, epidemiologic, and early warning surveillance systems which represents the largest body of information from private hospitals across Thailand. Hospitals and clinical laboratories have deployed automatic reporting mechanisms since 2010 and have effectively improved timeliness of laboratory data reporting. In April 2017, the capacity of near real-time influenza surveillance in BDMS was found to have a demonstrated and sustainable capability.

\section{Results}

From October 2010 to April 2017, a total of 482,789 subjects were tested and 86,110 (17.8\%) cases of influenza were identified. Of those who tested positive for influenza they were aged $<2$ years old $(4.6 \%)$, 2-4 year old ( $10.9 \%), 5-14$ years old $(29.8 \%), 15-49$ years old $(41.9 \%)$, $50-64$ years old $(8.3 \%)$ and $>65$ years old (3.7\%). Approximately $50 \%$ of subjects were male and female. Of these, $40,552(47.0 \%)$ were influenza type B, 31,412 (36.4\%) were influenza A unspecified subtype, 6,181 (7.2\%) were influenza A H1N1, 4,001 (4.6\%) were influenza A H3N2, 3,835 (4.4\%) were influenza A seasonal and 196 $(0.4 \%)$ were respiratory syncytial virus (RSV).
The number of influenza-positive specimens reported by the realtime influenza surveillance system were from week 40, 2015 to week 39,2016 . A total of 117,867 subjects were tested and 17,572 (14.91\%) cases tested positive for the influenza virus (Figure 1). Based on the long-term monitoring of collected information, this system can delineate the epidemiologic pattern of circulating viruses in near realtime manner, which clearly shows annual peaks in winter dominated by influenza subtype B in 2015-1016 season. This surveillance system helps to provide near real-time reporting, enabling rapid implementation of control measures for influenza outbreaks.

\section{Conclusions}

This surveillance system was the first real-time, daily reporting surveillance system to report on the largest data base of private hospitals in Thailand and provides timely reports and feedback to all stakeholders. It provides an important supplement to the routine influenza surveillance system in Thailand. This illustrates a high level of awareness and willingness among the BDMS hospital network to report emerging infectious diseases, and highlights the robust and sensitive nature of BDMS's surveillance system. This system demonstrates the flexibility of the surveillance systems in BDMS to evaluate to emerging infectious disease and major communicable diseases. Through participation in the Thailand influenza surveillance network, BDMS can more actively collaborate with national counterparts and use its expertise to strengthen global and regional surveillance capacity in Southeast Asia, in order to secure advances for a world safe and secure from infectious disease. Furthermore, this system can be quickly adapted and used to monitor future influenzas pandemics and other major outbreaks of respiratory infectious disease, including novel pathogens.

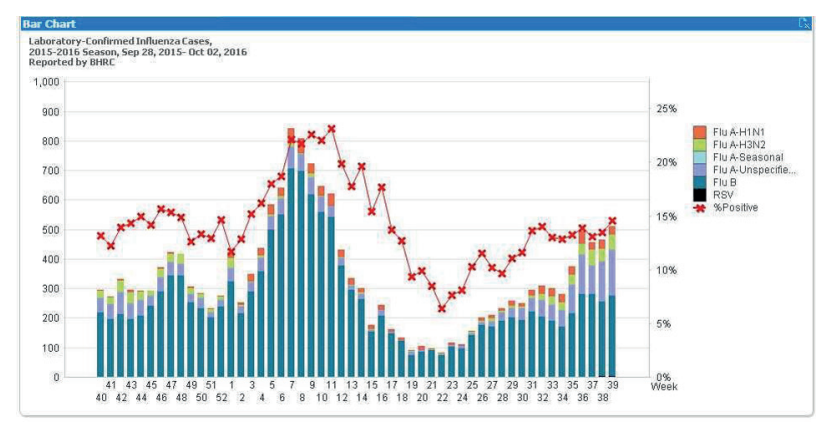

Weekly distribution of number of collected samples with influenza viruses, Bangkok Hospital Group, September 2015 to October 2016

\section{Keywords}

real-time surveillance system; influenza A H1N1; influenza A H3N2; influenza type B 
References

- Ghosh TS, Vogt RL. Active influenza surveillance at the local level: a model for local health agencies. Am J Public Health 2008; 98(2):213-5.

- Baumbach J, Mueller M, Smelser C, et al. Enhancement of influenza surveillance with aggregate rapid influenza test results: New Mexico, 2003-2007. Am J Public Health 2009;99 Suppl 2:S372-7.

- Dalton CB, Carlson SJ, Butler MT, et al. Building influenza surveillance pyramids in near real time, Australia. Emerg Infect Dis 2013;19(11):1863-5.

\section{*Phunlerd Piyaraj}

E-mail: p_phunlerd@yahoo.com 